The Astrophysical Journal, 322:706-720, 1987 November 15

(C) 1987. The American Astronomical Society. All rights reserved. Printed in U.S.A.

\title{
A COMPOSITE CO SURVEY OF THE ENTIRE MILKY WAY
}

\author{
T. M. Dame, ${ }^{1}$ H. Ungerechts, ${ }^{1}$ R. S. Cohen, ${ }^{2}$ E. J. De Geus, ${ }^{3}$ I. A. Grenier, ${ }^{4}$ \\ J. May, ${ }^{5}$ D. C. MurPhy, ${ }^{6}$ L.-Å. Nyman, ${ }^{1}$ and P. Thaddeus ${ }^{1}$ \\ Received 1987 February 4; accepted 1987 May 13
}

\begin{abstract}
Large-scale CO surveys of molecular clouds in the Milky Way, first undertaken with a $1.2 \mathrm{~m}$ telescope in New York City, have been extended along the entire Galactic plane with a similar instrument on Cerro Tololo, Chile. By combining five large unbiased surveys of segments of the Galactic plane with 11 surveys of particular local clouds (e.g., the Taurus dark clouds), a composite map $10^{\circ}-30^{\circ}$ wide in latitude has been obtained at an angular resolution of 0.5 . It is clear from a comparison with dark nebulae, and from the $I R A S$ far-infrared and COS B gamma-ray surveys that little molecular gas lies beyond the boundary of our composite map. The full composite survey contains more than 31,000 spectra and fully samples $\sim 7700$ deg $^{2}$, nearly a fifth of the entire sky. An $l, v$ diagram has been produced for a subset of the survey, the data from a strip 6.5 wide in $b$ centered on the Galactic equator.

The main structural features of the molecular Galaxy are readily apparent at an angular resolution of 0.5 . The molecular clouds in the inner spiral arms of the Galaxy, the so-called molecular ring, appear as an intense ridge of emission $\sim 2^{\circ}$ wide in $b$ extending $\sim 60^{\circ}$ on either side of the Galactic center. The $l$, $v$ diagram reveals large departures from axisymmetry both in the molecular ring and elsewhere; most striking are the large arcs of the Carina and Perseus spiral arms. The local (low-velocity) emission mainly follows Gould's Belt of OB stars, gas, and dust: large clouds in Lupus, Ophiuchus, and Aquila mark the Belt's positive-latitude extension, while the Taurus and Orion clouds, directly opposite in the sky, lie at the negative-latitude extension.

The complete longitude and the wide latitude coverage of the composite survey permits, for the first time, a fairly complete determination of the distribution of molecular clouds near the Sun. It has long been suspected from the distribution of dark clouds and rifts that local molecular clouds are more common in the northern Milky Way than in the southern, and the composite survey confirms this quantitatively: the molecular mass within $1 \mathrm{kpc}$ is 4 times greater in the first and second quadrants than in the third and fourth. Furthermore, nearly all the clouds within $1 \mathrm{kpc}$ in the first and fourth quadrants apparently lie on a fairly straight ridge more than $1 \mathrm{kpc}$ long which may trace the inner edge of the Local spiral arm. The measured rms dispersion about the Galactic plane of the local molecular gas, $74 \mathrm{pc}$, corresponds to a Gaussian with half-thickness at half-intensity of $87 \mathrm{pc}$. The mean surface density of molecular gas within $1 \mathrm{kpc}$ of the Sun is $1.3 M_{\odot} \mathrm{pc}^{-2}$; on the assumption of a Gaussian $z$ distribution with a half-thickness of $87 \mathrm{pc}$, the mean midplane density at the solar circle is $0.0068 \mathrm{M}_{\odot} \mathrm{pc}^{-3}$, or $0.10 \mathrm{H}_{2} \mathrm{~cm}^{-3}$.

Subject headings: galaxies: The Galaxy — galaxies: structure — interstellar: molecules
\end{abstract}

\section{INTRODUCTION}

In spite of great advances in instrumentation, radio astronomical surveys of molecular clouds in the Milky Way since the discovery of the ubiquitous tracer molecule CO in 1970 have not proceeded as systematically or as rapidly as the early $21 \mathrm{~cm}$ surveys a generation ago. A year after Ewen and Purcell's (1951) detection of the $21 \mathrm{~cm}$ line, Christiansen and Hindman (1952) completed a preliminary large-scale $21 \mathrm{~cm}$ survey covering $\pm 50^{\circ}$ of Galactic latitude over $270^{\circ}$ of Galactic longitude; their angular resolution was poor but their sampling was good, and most of the important large-scale $21 \mathrm{~cm}$ features, including the Galactic center, Vela, and Cygnus $X$ regions along the plane and the high- $z$ extensions in Ophiuchus and Taurus are evident in their maps. Within a few years this preliminary work was followed by the Leiden survey of the northern sky (Muller and Westerhout 1957) and the Sydney survey of the southern

\footnotetext{
${ }^{1}$ Harvard-Smithsonian Center for Astrophysics.

${ }^{2}$ Department of Physics, Columbia University.

${ }^{3}$ Sterrewacht, Leiden, The Netherlands.

${ }^{4}$ Service d'Astrophysique, CEN Saclay, France.

${ }^{5}$ Departamento de Astronomía, Universidad de Chile.

6 European Southern Observatory.
}

sky (Kerr, Hindman, and Gum 1959); done with inexpensive antennas dedicated to hydrogen-line work for long periods, these completely mapped at $\sim 2^{\circ}$ angular resolution the distant spiral arms of the Milky Way around the Galactic equator and followed local gas to fairly high latitudes $\left( \pm 10^{\circ}\right)$, establishing a secure foundation for subsequent higher resolution observations with larger antennas and interferometers that continue to the present day.

The first Galactic CO surveys, in contrast, were confined to a thin strip along the Galactic equator in the northern hemisphere and only a minute fraction of the molecular gas in the Galaxy was actually observed (Scoville and Solomon 1975; Gordon and Burton 1976; Cohen and Thaddeus 1977). Owing to the small number of millimeter-wave instruments, the small beamwidths of most $\left(\sim 1^{\prime}\right)$, and the close association of $\mathrm{CO}$ clouds with a variety of cataloged Population I objects, the progress of unbiased $\mathrm{CO}$ surveys has been slow, and, until now, no survey comparable in coverage and sampling to the Sydney-Leiden surveys had been undertaken.

The Columbia millimeter-wave telescope in New York and its Twin instrument in Chile were designed to carry out $\mathrm{CO}$ surveys of molecular clouds north and south in the spirit of the Sydney-Leiden surveys. The $1.2 \mathrm{~m}$ antennas of these telescopes 
yield a beamwidth of 8.7 at the frequency of the $\mathrm{CO} 1 \rightarrow 0$ line, equivalent to a $100 \mathrm{~m}$ antenna operating at $21 \mathrm{~cm}$. With these two telescopes it has been possible to produce well-sampled surveys of the distant inner Galaxy and of specific local clouds; but to obtain the wide-latitude coverage required for comparison with the IRAS and $\operatorname{COS} B$ surveys in a reasonable amount of time, a lower resolution, closer to that of the Sydney-Leiden surveys, was required.

Lower resolution was achieved not by stopping down the antenna (an inconvenient procedure with a Cassegrain) but by the simple expedient of sequentially stepping through a square array of points about a beamwidth apart during a single observation and summing the spectra obtained. Information is acquired at essentially the same rate as with a smaller antenna whose beam matches the array in size, and, implemented entirely by computer software, the procedure has the practical merit of allowing the telescope to revert at any time to observations at full resolution without modification of feed or antenna.

This technique was first used to map the first Galactic quadrant over more than $10^{\circ}$ of Galactic latitude (Dame and Thaddeus 1985); an $8 \times 8$ array was scanned to yield an angular resolution of $1^{\circ}$. Since the completion of this survey in 1980 , the array scanning technique has been applied to a number of different surveys that now cover most of the Milky Way in a somewhat irregular quilt. In this paper we present a synoptic view of the entire Milky Way produced from a synthesis of five major Galactic plane surveys and 11 surveys of particular regions, such as the Taurus dark nebulae. Most are at an angular resolution of $\frac{1}{2}^{\circ}$, the rest smoothed to this resolution. The total composite survey contains more than 31,000 spectra and fully samples $\sim 7700 \mathrm{deg}^{2}$, nearly a fifth of the entire sky. We will not attempt here either to derive the large-scale distribution of molecular clouds in the Galaxy or to Study particular regions, as that is being done elsewhere; we do derive, however, some important parameters not previously very well measured, in particular, the mean density and scale height of molecular gas within $1 \mathrm{kpc}$ of the Sun, and we attempt to locate the main molecular concentrations within this region on the Galactic plane.

The next section ( $\S$ II) describes the two telescopes, the observing techniques used for the individual surveys, and how these were reconciled and joined together. In $\S$ III the composite survey is presented as a spatial map integrated over velocity and as a longitude-velocity map integrated over latitude; individual clouds and large-scale features of the maps are briefly discussed. In $\S$ IV the distribution and properties of molecular clouds within $1 \mathrm{kpc}$ of the Sun are investigated and in $\S \mathrm{V}$ we demonstrate by a comparison with other Population I tracers that the composite survey probably provides a nearly complete inventory of nearby molecular clouds.

\section{OBSERVATIONS AND ANALYSIS}

\section{a) Observations}

The Chile telescope is a close copy of that in New York, and the two together provide the first means of observing molecular clouds over the entire sky with similar instrumentation. Both telescopes have fast Cassegrain antennas with a primary aperture of $1.2 \mathrm{~m}$ and beamwidths of $8.7 \pm 0.1$ at $115 \mathrm{GHz}$, the frequency of the $1 \rightarrow 0$ rotational transition of $\mathrm{CO}$ used as the standard tracer of molecular clouds. The main instrumental differences were in receiver sensitivity and spectrometer resolution, and these were easily reconciled in data taking (by adjusting integration times) and data analysis (by smoothing) and are not an important source of inhomogeneity in the final composite survey. For the work described here, two superheterodyne receivers were used in succession on the New York telescope, the first with an uncooled Schottky diode mixer (Cong, Kerr, and Mattauch 1979) and a single-sideband receiver noise temperature of $900 \mathrm{~K}$, the second, installed in the summer of 1983 , with an extremely sensitive liquid heliumcooled SIS mixer (Pan 1984) and a single-sideband temperature of $95 \mathrm{~K}$. On the Chile telescope an inexpensive but rugged and sensitive liquid nitrogen-cooled Schottky diode receiver with a noise temperature of $385 \mathrm{~K}$ was used. For spectrometers, both instruments were equipped with 256 channel filter banks copied with minor improvements from an NRAO design (Mauzy 1974), that in New York with a frequency resolution of $250 \mathrm{kHz}$, or $0.65 \mathrm{~km} \mathrm{~s}^{-1}$ at $115 \mathrm{GHz}$, that in Chile with a resolution of $500 \mathrm{kHz}$, or $1.3 \mathrm{~km} \mathrm{~s}^{-1}$ at 115 $\mathrm{GHz}$; a second filter bank with $100 \mathrm{kHz}$ resolution was used on the Chile telescope for two small regions.

Spectral line intensities were calibrated and corrected for atmospheric absorption by briefly observing before each integration a room-temperature chopper wheel in front of the feed horn (Penzias and Burrus 1973). The effective temperature and opacity of atmospheric water vapor were determined every 6 $\mathrm{hr}$, or more often during weather changes, by antenna tipping. Intensities were further corrected for main beam efficiency to yield radiation temperatures that agree to within 5\% with all data from the New York and Chile telescopes reported since 1980. In particular, the intensities reported here agree with those of the Wide-Latitude survey of Dame and Thaddeus (1985), used by Bloemen et al. (1986) to determine the ratio of velocity-integrated $\mathrm{CO}$ intensity to $\mathrm{H}_{2}$ column density. Although a recent reevaluation of the beam efficiencies of the New York and Chile telescopes by Bronfman et al. (1987) suggests that this intensity scale may be low by $\sim 20 \%$ in absolute units, we retain it here for consistency with the $\mathrm{CO}$ mass calibration.

Most of the data were taken by position switching against one to three reference ("OFF") positions with a period of $30 \mathrm{~s}$; such spectra toward emission-free regions typically were quite flat, so only linear baselines were removed. In some regions well off the Galactic equator, however, with only one or occasionally two low-velocity CO lines (e.g., the Taurus dark clouds), frequency switching by 10 to $20 \mathrm{MHz}$ was used instead to increase the speed of data acquisition; higher order baselines were generally required to fit such spectra, but, because the lines in such regions are narrow, integrated line intensities were insensitive to the order of the fit.

Within $\sim 5^{\circ}$ of the Galactic center intense emission extends over more than the $332 \mathrm{~km} \mathrm{~s}^{-1}$ bandwidth of the spectrometer on the Chile telescope, so it was necessary there to observe each direction twice, shifting the spectrometer by $\sim 100 \mathrm{~km}$ $\mathrm{s}^{-1}$ and joining the resultant spectra (Nyman et al. 1987a).

The New York telescope has recently been described in detail by Cohen, Dame, and Thaddeus (1986), and the Chile telescope by Cohen (1983) and Grabelsky (1985); the calibration procedures used on both telescopes are discussed by Cohen, Dame, and Thaddeus (1986), and a detailed comparison of their calibrations is given by Bronfman et al. (1987).

\section{b) Synthesis of the Data}

The New York and Chile telescopes have now mapped a strip $10^{\circ}-20^{\circ}$ wide in Galactic latitude around the entire 
Galactic plane, and nearly all of the large, nearby regions of optical obscuration and star formation at higher latitude as well. These surveys, five large Galactic plane surveys, and 11 others are summarized in Table 1; the sky coverage of each is shown in Figure i. These surveys were generally fully sampled at an angular resolution of 0.5 , but some differences in coordinate systems, sampling patterns, beamwidths, and velocity resolution exist. We discuss below the general procedures used to combine these surveys to produce a single velocityintegrated spatial map and a Galactic latitude-integrated longitude-velocity map of the entire Milky Way. Specific details on the treatment of individual surveys can be found in the notes to Table $\mathbf{i}$.

The individual surveys provide nearly complete velocity coverage of Galactic emission in all observed directions. To produce the composite spatial map, the spectra at low latitudes in the first and fourth quadrants were integrated over the full bandwidth of the spectrometer to cover all emission within the solar circle and most beyond. (Between $l=12^{\circ}$ and $l=60^{\circ}$, the velocity coverage was not adequate to observe all material beyond the solar circle, but, owing to the rapid falloff in density of molecular clouds beyond the solar circle [Solomon, Stark, and Sanders 1983; Grabelsky et al. 1987; Kutner and Mead 1985] and the large distance of this region from the Sun, its inclusion probably would have changed the map only very slightly.) In the second and third quadrants, the spectrometer bandwidths were adequate to cover all velocities where $21 \mathrm{~cm}$ emission is significant, and the data were integrated over all detected emission. More than $3^{\circ}$ from the Galactic equator the data were generally integrated only over the low-velocity interval where emission was detected or expected.

Several surveys (as indicated in Table 1) required special treatment: local surveys done in equatorial coordinates or on an $l, b$ grid shifted from the main map grid were added to the main map by numerical interpolation, while others taken with an angular resolution of $\frac{1}{8}^{\circ}$ or $\frac{1}{4}^{\circ}$ were smoothed to the $\frac{1}{2}^{\circ}$ resolution of the composite map. In regions covered by more than one survey, that with the lowest noise was used.
The noise levels of the surveys varied between 0.1 and $0.35 \mathrm{~K}$ rms per channel. The noise in the composite spatial map therefore varies somewhat from region to region, depending on channel width, beamwidth, and spectral baseline fit of individual surveys, and, further, on the velocity-integration range and the amount of interpolation and smoothing applied. The lowest contour level for the composite map, $5 \mathrm{~K} \mathrm{~km} \mathrm{~s}^{-1}$, lies at about 2.5 times the highest noise level. A contour map of the negative values in the composite spatial map verified that, except in a small region near the plane between $l=128^{\circ}$ and $140^{\circ}$ (perhaps contaminated by a bad ofF position), the number of positions with integrated temperature below $-5 \mathrm{~K}$ $\mathrm{km} \mathrm{s}^{-1}$ was consistent with an rms noise everywhere in the map of less than $\sim 2 \mathrm{~K} \mathrm{~km} \mathrm{~s}^{-1}$.

Compared with the spatial map, the longitude-velocity map integrated over \pm 3.25 in latitude, involving only the Galactic plane surveys (numbers 2, 4, 9, 11, and 13 in Table 1), was relatively simple to produce. After integration over latitude, the northern surveys ( 2 and 4 ) were smoothed in velocity to a resolution of $1.3 \mathrm{~km} \mathrm{~s}^{-1}$ to match the resolution of the southern surveys, and every second velocity channel from the northern surveys was used for the composite map. The latitude integration range includes essentially all material outside the Local spiral arm.

\section{THE COMPOSITE MAPS}

The composite spatial map is shown in contour form in the Figure 2 foldout (Plate 5). The burned out region of intense emission near the Galactic plane is shown clearly with more widely spaced contours in the maps in the lower portion of the figure. The Figure 3 foldout (Plate 6) represents the composite longitude-velocity map, integrated over \pm 3.25 in latitude and covering $360^{\circ}$ of Galactic longitude; Figure 4 is a blowup of the Galactic center region, adding the small amount of highvelocity emission clipped in Figure 3. In Figure 5 the survey is shown, integrated over both radial velocity and Galactic latitude, as a function of Galactic longitude.

The composite maps are discussed below starting from the

TABLE 1

INDIVIDUAL SURVEYS

\begin{tabular}{|c|c|c|c|c|c|c|c|c|}
\hline No. & Region & $l_{\min }$ & $l_{\max }$ & $b_{\min }$ & $b_{\max }$ & Telescope & Note & Ref. \\
\hline $1 \ldots \ldots \ldots$ & Oph-Sgr & $8^{\circ}$ & $40^{\circ}$ & $9^{\circ}$ & $24^{\circ}$ & N1 & 1 & 1 \\
\hline $2 \ldots \ldots \ldots$ & 1st Quad & 12 & 100 & -10 & 10 & N2 & & 2 \\
\hline $3 \ldots \ldots \ldots$ & Aquila South & 27 & 40 & -21 & -10 & N1 & 1 & 3 \\
\hline $4 \ldots \ldots \ldots$ & 2nd Quad & 98 & 180 & -4 & 10 & N1 & & 4 \\
\hline $5 \ldots \ldots \ldots$ & Cepheus & 99 & 143 & 8 & 22 & N2 & & 5 \\
\hline $6 \ldots \ldots \ldots$ & Taurus & 152 & 180 & -25 & -3 & N2 & 2 & 6 \\
\hline $7 \ldots \ldots \ldots$ & Anticenter & 175 & 210 & -9 & 7 & N1 & & 7 \\
\hline $8 \ldots \ldots \ldots$ & Orion & 180 & 225 & -25 & 5 & N1 & 2,3 & 8 \\
\hline $9 \ldots \ldots \ldots$ & 3rd Quad & 180 & 279 & -5 & 5 & $\mathrm{C}$ & & 9 \\
\hline $10 \ldots \ldots \ldots$ & Carina-deep & 270 & 300 & -2 & 2 & $\mathrm{C}$ & 3 & 10 \\
\hline $11 \ldots \ldots \ldots$ & Carina-local & 270 & 300 & -5 & 5 & $\mathrm{C}$ & & 10 \\
\hline $12 \ldots \ldots \ldots$ & Chamaeleon & 292 & 308 & -20 & -12 & $\mathrm{C}$ & 2,3 & 11 \\
\hline $13 \ldots \ldots \ldots$ & 4th Quad and Ctr & 300 & 15 & -8 & 8 & $\mathrm{C}$ & & 12 \\
\hline $14 \ldots \ldots \ldots$ & Lupus & 333 & 351 & 1 & 23 & $\mathrm{C}$ & & 13 \\
\hline $15 \ldots \ldots \ldots$ & Ophiuchus & 344 & 4 & 7 & 25 & $\mathrm{C}$ & 4,5 & 14 \\
\hline $16 \ldots \ldots \ldots$ & $\mathrm{R} \mathrm{CrA}$ & 358 & 4 & -24 & -16 & $\mathrm{C}$ & 4,5 & 14 \\
\hline
\end{tabular}

TELESCOPE. N1 = NY with Schottky receiver; N2 = NY with SIS receiver $; \mathrm{C}=\mathrm{Chile}$.

NoTES.- (1) Sampling grid shifted by $0.25 ;(2)$ transformed from equatorial to Galactic coordinate grid; (3) smoothed from 0.125 to 0.5 angular resolution; (4) Smoothed from 0.25 to 0.5 angular resolution; (5) $0.26 \mathrm{~km}$ $\mathrm{s}^{-1}$ velocity resolution.

REFERENCES.-(1) Lebrun and Huang 1984; (2) Dame, Grenier, and Thaddeus 1987; (3) Lebrun 1986; (4) Cohen et al. 1980 and subsequent observations by J. Brock; (5) Grenier, Dame, and Thaddeus 1987; (6) Ungerechts and Thaddeus 1987; (7) Huang 1985;(8) Maddalena et al. 1986; (9) Murphy 1985;(10) Grabelsky et al. 1987; (11) Koprucu, Cohen, and Thaddeus 1987; (12) Nyman et al. 1987a; (13) Murphy, Cohen, and May 1986;(14) de Geus 1987. 


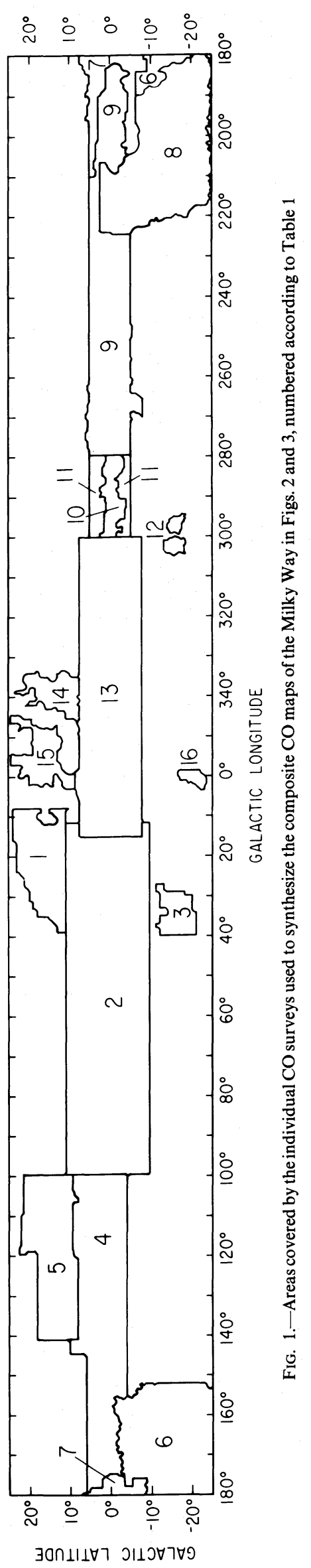

(C) American Astronomical Society - Provided by the NASA Astrophysics Data System 


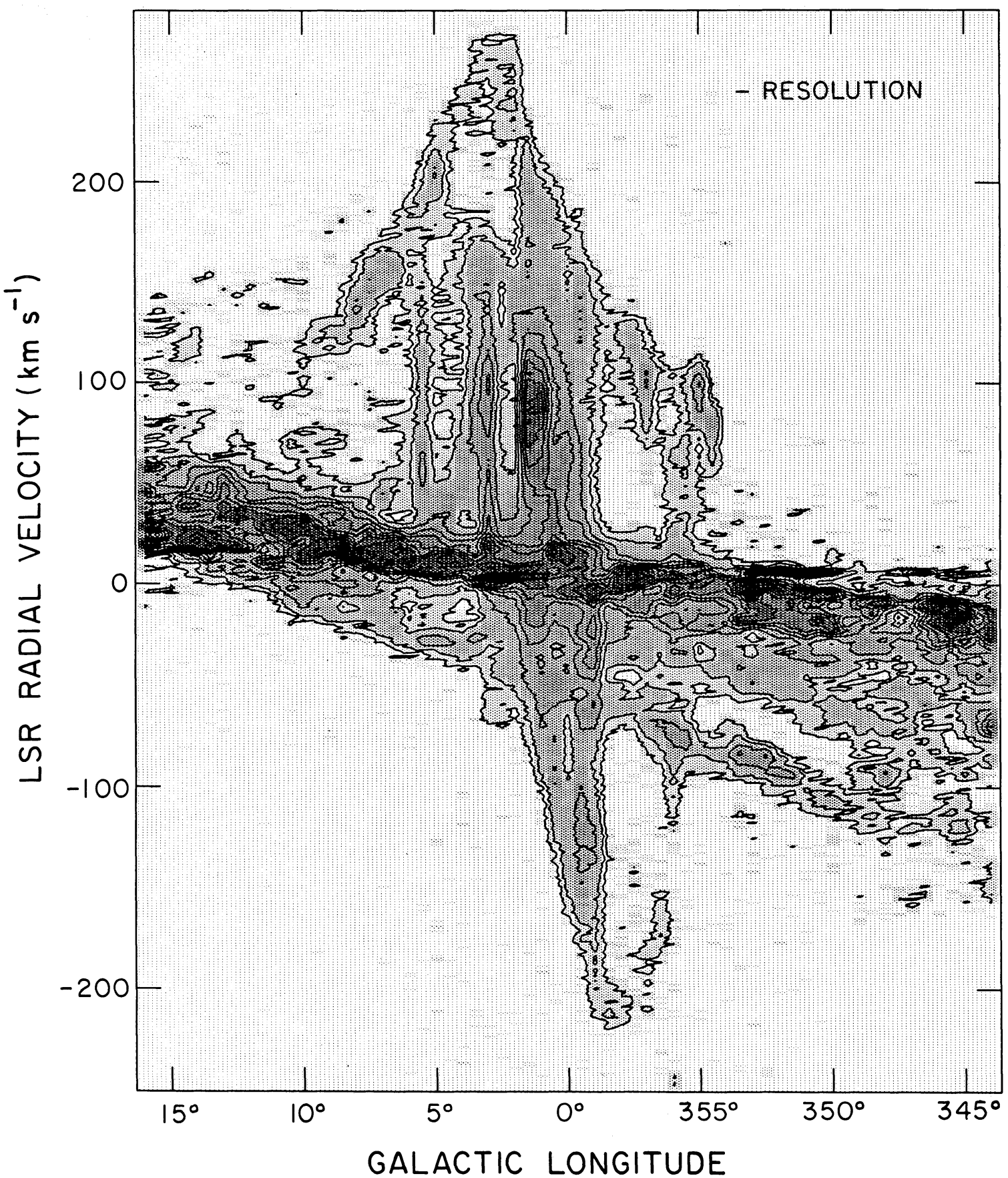

Fig. 4.-Expanded version of the Galactic center region from Fig. 3, covering the full velocity extent of the emission. Contours are the same as in Fig. 3, except for an additional contour at $0.4 \mathrm{~K} \mathrm{deg}$.

far left in Figures 2 and 3 (second quadrant), through the Galactic center, to the far right (third quadrant), and back to the starting point at $l=180^{\circ}$. We note major clouds and regions and cite sources of the data as well as previous surveys carried out with the New York and Chile telescopes. In Figure 6 cloud complexes and other large-scale features are labeled by their common names, taken generally from constellations or from well-known associated objects.

\section{a) Individual Regions}

The dark clouds in Taurus and Perseus well below the Galactic plane between $l=155^{\circ}$ and $180^{\circ}$ were observed and discussed by Ungerechts and Thaddeus (1987), who identified three major molecular clouds, one at $\sim 140 \mathrm{pc}$ associated with the dark nebulae in Taurus and Auriga, a second at $\sim 350 \mathrm{pc}$ associated with IC 348 and NGC 1333, and a third at about the same distance associated with the California nebula (NGC 1499) and NGC 1579. The second and third clouds mentioned lie on either side of the Per OB2 association, also at $350 \mathrm{pc}$ (Fig. 6).

The rest of the second Galactic quadrant is characterized by widespread filamentary emission lying almost entirely in two velocity intervals: between 0 and $-10 \mathrm{~km} \mathrm{~s}^{-1}$ from the Local spiral arm, and between -40 and $-60 \mathrm{~km} \mathrm{~s}^{-1}$ from the Perseus arm (Fig. 3); the three largest molecular complexes associated with the Perseus arm in the second quadrant, which 


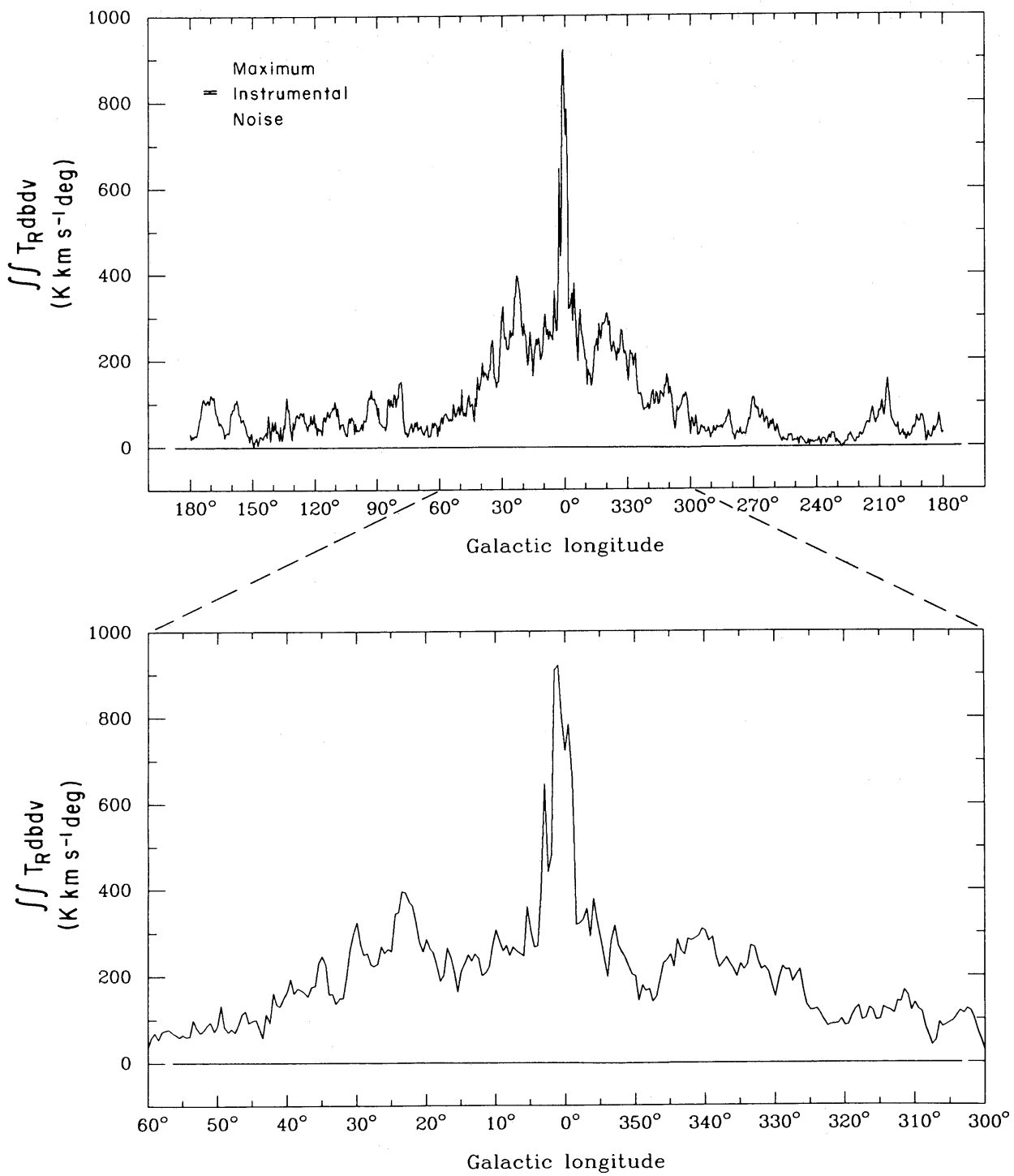

Fig. 5.-Longitude profile of the composite survey integrated over all velocities and over latitude. The instrumental noise varies from 2 to $6 \mathrm{~K} \mathrm{~km} \mathrm{~s} \mathrm{~s}^{-1}$ deg. Most of the apparent noise is not instrumental-it is the "shot noise" in the distribution of molecular clouds. The lower curve is simply a blowup of the central $120^{\circ}$ of longitude.

we designate NGC 7538, W3, and S235, are indicated in Figure 6. This region was discussed both by Cohen et al. (1980) and Dame et al. (1986), except for the Cepheus region, observed by Grenier, Dame, and Thaddeus (1987).

The first quadrant survey of Dame, Grenier, and Thaddeus (1987) covers intense emission from the inner Galaxy spiral arms and the local dark clouds that form the Great Rift in the Milky Way. Although few individual clouds can be distinguished in Figure 2, two fairly local dark nebulae are visible, Cyg OB7 and the Aquila Rift, as well as three quite distant and massive molecular complexes, W51, W44, and M17, all identified in Figure 6. Previously, Dame and Thaddeus (1985) mapped most of this region at $1^{\circ}$ spatial resolution and showed that with the information provided by the $\mathrm{CO}$ radial velocities it was possible to resolve the Great Rift into 10 distinct molecular clouds lying over a wide range of distances, 200 to $2300 \mathrm{pc}$.

The Ophiuchus-Sagittarius region above the Aquila Rift (region 1 in Fig. 1) was studied by Lebrun and Huang (1984) because the optical opacity (Heiles 1976; Burstein and Heiles
1978) and diffuse $\gamma$-ray emission (Lebrun and Paul 1983) there are greater than expected from $21 \mathrm{~cm}$ observations. They discovered an appreciable amount of cold molecular gas, some so faint in CO that it falls below the lowest contour of Figure 2. South of the Aquila Rift is a region with a similar H I deficiency (region 3 from Fig. 1), but Lebrun (1986) found only a few very small clouds there, all below the lowest contour of Figure 2.

Well above the Galactic plane near the center of the map lie the similar, possibly related $\rho$ Oph and Lupus molecular cloud complexes at 100 to $200 \mathrm{pc}$, each with masses of a few $10^{4} M_{\odot}$. The $\rho$ Oph clouds, which contain one of the nearest, most closely studied regions of star formation, were recently observed by de Geus (1987) with the Chile Telescope at full resolution but with $\frac{1}{4}^{\circ}$ spacing; an earlier, preliminary $\frac{1}{2}^{\circ}$ resolution survey had been carried out from New York by Bronfman (1980). The Lupus clouds, mapped from Chile (Murphy 1985; Murphy, Cohen, and May 1986), contain a large number of $\mathrm{T}$ Tauri stars sometimes referred to as the Lupus T association (Schwartz 1977). 

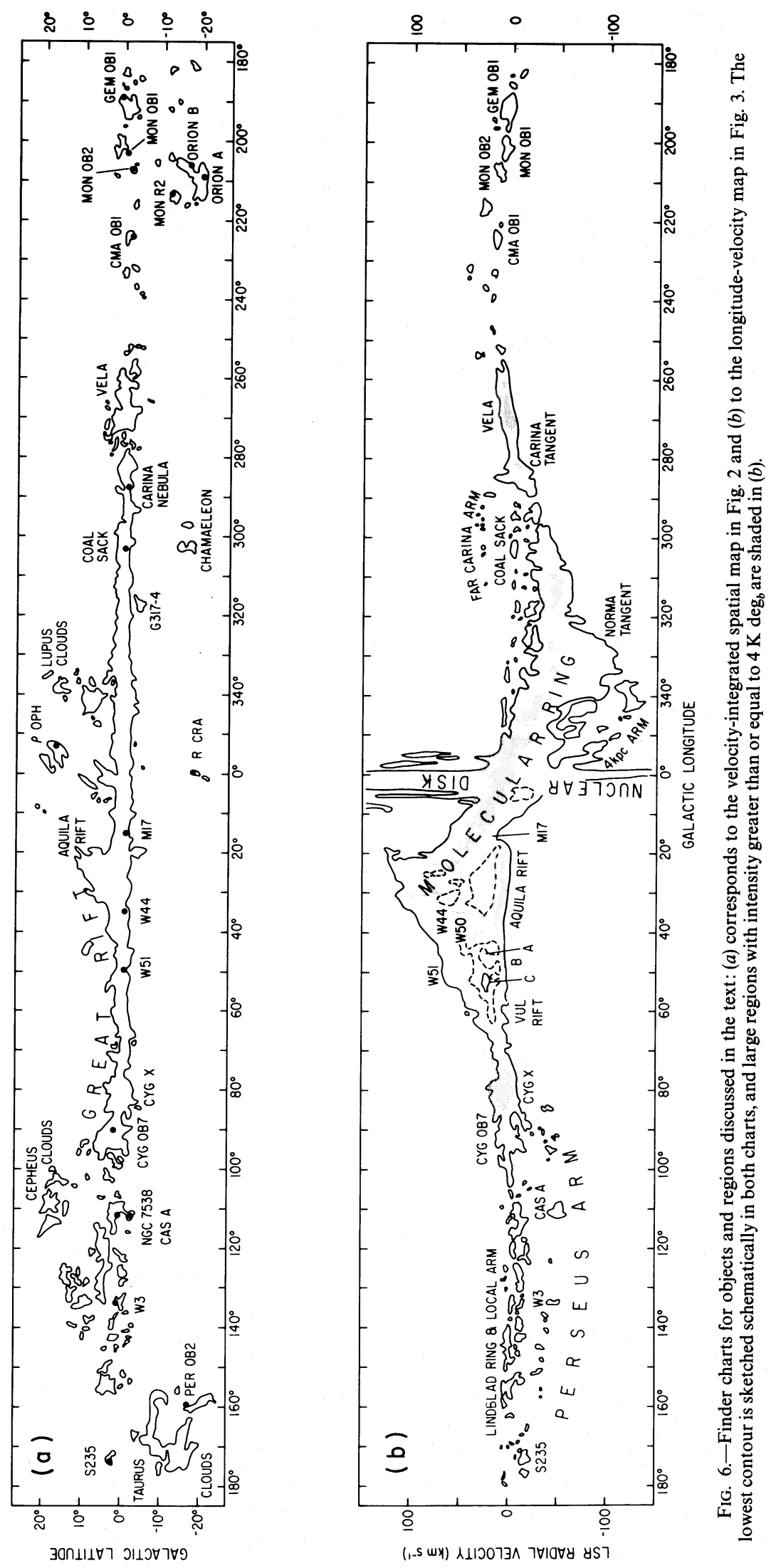
Far below the plane near the middle of the map lie the small, isolated R CrA and Chamaeleon clouds, well apart from the dark clouds and other young objects in Gould's Belt, which at this longitude is above the plane (see $\S$ III $b$ ). The R CrA cloud was mapped at $\frac{1}{4}^{\circ}$ angular resolution from Chile by de Geus (1987); CO observations toward the young, luminous, emission-line star R CrA were previously reported by Loren, Peters, and Vanden Bout (1974). Most of the Chamaeleon region was mapped at full resolution from Chile by Koprucu, Cohen, and Thaddeus (1987); Keto and Myers (1986) observed three small clouds on the fringe of the main star-forming cloud.

The fourth-quadrant survey of Nyman et al. (1987a), covering the intense emission from the Galactic center and the inner spiral arms, has a lower noise level $(0.1 \mathrm{~K} \mathrm{rms})$ than most others; essentially all features within its boundaries in Figure 2, even those so small as to be taken as noise, are real molecular clouds. For example, the lower two of the three small features lying above the plane near $l=325^{\circ}$ are intense regions of a large $\left(10^{5} M_{\odot}\right)$ cloud that Nyman et al. $(1987 b)$ found to lie $\sim 250 \mathrm{pc}$ above the Galactic plane, nearly 3 times the molecular cloud half-thickness in $z$. Its situation above a conspicuous hole in the longitude-velocity distribution of $\mathrm{CO}$ in the plane suggests that both the large displacement of the cloud and the hole itself may be due to a single cause, possibly multiple supernova explosions and stellar winds. In contrast to the first quadrant, blanketed by nearby dark clouds, the fourth quadrant is comparatively free of low-velocity emission (Fig. 3), with the conspicuous exception of the Coalsack, identified in Figure 6 even though in the spatial map (Fig. 2) it is badly confused with more intense background emission from the inner Galaxy.

The $\mathrm{CO}$ emission toward the Galactic center extends over a very wide velocity range, roughly $\pm 250 \mathrm{~km} \mathrm{~s}^{-1}$ (Fig. 4), and the integrated intensity in Figure 2 reaches $948 \mathrm{~K} \mathrm{~km} \mathrm{~s}^{-1}$, about 4 times the highest intensities found elsewhere along the Galactic equator (see also Fig. 5). Among the most striking features in this region are the objects near $l=3^{\circ}$ and $5^{\circ}$ which stand out in the lower panels of Figure 2 due to their high integrated intensities and in Figure 4 due to their extraordinary velocity widths $\left(\sim 100 \mathrm{~km} \mathrm{~s}^{-1}\right)$. Their $\mathrm{CO}$ luminosities and velocity widths imply kinetic energies of order $10^{54} \mathrm{ergs}$, comparable to those of the $3 \mathrm{kpc}$ and $135 \mathrm{~km} \mathrm{~s}^{-1}$ expanding arms (Bitran, Thaddeus, and Cohen 1987).

The Carina nebula lies fairly near the tangent point at $280^{\circ}$ of the Carina spiral arm, one of the best defined structural features of the Galaxy in molecular clouds. The tangent is intense in $\mathrm{CO}$, while just beyond it (near $l \approx 275^{\circ}$ ) the CO intensity drops abruptly (Fig. 5). In the longitude-velocity map (Fig. 3 ), the Carina emission clearly shows the loop characteristic of a Galactic spiral arm. Grabelsky (1985) mapped this region at $\frac{1}{2}^{\circ}$ angular resolution and the inner $2^{\circ}$ to $3^{\circ}$ in latitude at full resolution. The large-scale properties of the Carina arm are discussed by Cohen et al. (1985) and Grabelsky et al. (1987).

A large part of the third Galactic quadrant, from $210^{\circ}$ to $279^{\circ}$, including the rich region in Vela centered at about $265^{\circ}$, was surveyed by May, Murphy, and Thaddeus (1987) and Murphy (1985) at $\frac{1}{2}^{\circ}$ resolution. In Vela, Murphy identified at least four massive $\left(\sim 10^{5} M_{\odot}\right)$ hot clouds between $800 \mathrm{pc}$ and $2400 \mathrm{pc}$ from the Sun, as well as closer gas, possibly related to the "Vela Sheet" of Eggen (1980), at approximately the distance of the Vela pulsar and the Gum nebula, $\sim 400 \mathrm{pc}$. Spread along the plane over the rest of the third quadrant are several well defined, star-forming clouds, one of them associated with the Rosette nebula (Mon OB2; Blitz and Thaddeus 1980), another with the Cone Nebula (Mon OB1; Blitz 1978). The quite massive $\left(\sim 10^{6} M_{\odot}\right)$ but strangely cold, quiescent cloud studied by Maddalena and Thaddeus (1985) is just below the plane between the CMa OB1 and Mon OB2 clouds. Overlapping sections of the region $l=180^{\circ}$ to $230^{\circ}$ have been observed at $\frac{1}{2}^{\circ}$ resolution from New York by Huang (1985) and Maddalena and Thaddeus (1985) and from Chile by Murphy (1985).

Three of the most extensively studied molecular clouds, those associated with the Orion Nebula, NGC 2024 (Orion B), and Mon R2, are conspicuous well below the plane near $l \sim 210^{\circ}$. This region, mapped and discussed in detail by Maddalena et al. (1986), also contains numerous smaller clouds and two interesting molecular filaments, each more than $10^{\circ}$ long and typically $\frac{1}{2}^{\circ}$ wide, which reach toward the Galactic plane. These filaments, barely resolved and lying mainly below the lowest contour, are not visible in Figure 2.

\section{b) Large-Scale Structure}

The intense ridge of $\mathrm{CO}$ emission along the central $120^{\circ}$ of the Galactic equator is the most prominent feature in Figure 2. As the longitude-velocity map (Fig. 3) shows, the high intensity of the emission results from integration over many clouds along the line of sight, most of them lying in the inner Galaxy spiral arms or in the region of the Galactic center. The rapid falloff in density of molecular clouds beyond the solar circle is evident when the intensity of this ridge is compared with emission at low latitudes in the second and third quadrants, where nonlocal outer Galaxy clouds appear.

Excluding the inner-Galaxy ridge, most of the emission in the spatial map (Fig. 2) arises from clouds within $\sim 1 \mathrm{kpc}$. Except in small regions of severe velocity crowding near $l=0^{\circ}$, $90^{\circ}, 180^{\circ}$, and $270^{\circ}$, these local clouds clearly are separated in velocity from the more distant ones (see Fig. 3): in the first and third quadrants they generally lie between -10 and $20 \mathrm{~km} \mathrm{~s}^{-1}$, while in the second and fourth they fall between -20 and 10 $\mathrm{km} \mathrm{s}^{-1}$. The large clouds in Figure 2 more than $3^{\circ}$ from the plane, excluded from the longitude-velocity map (Fig. 3), also fall within these velocity ranges.

A striking aspect of the local molecular cloud distribution is the much higher density of clouds in the northern half of the Milky Way $\left(l=0^{\circ}\right.$ to $\left.180^{\circ}\right)$ than in the southern $\left(180^{\circ}\right.$ to $\left.360^{\circ}\right)$. This asymmetry stands out in the longitude-velocity map (Fig. $3)$, even though much of the local emission in the northern Milky Way lies beyond $\pm 3^{\circ}$ in latitude and is therefore excluded. The preponderance of nearby dark clouds in the north is very evident on large-scale optical surveys, the Milky Way from Sagittarius to Cepheus being divided by the Great Rift of optical obscuration (Dame and Thaddeus 1985; Feitzinger and Stüwe 1986a) and the region beyond, from Cepheus to the anticenter, covered by a complex system of filamentary dark clouds. Seen clearly in Figure 2 between $l=100^{\circ}$ and $180^{\circ}$, these filamentary clouds have been called the Northern Dark Cloud System by Schlosser and Görnandt (1984), who suggested they constitute a coherent structure. Figure 2 lends some support to this idea, since the extensive, filamentary emission of the region sharply contrasts with most other regions of the map, the fourth quadrant in particular.

One large-scale structure evident in Figure 2 is associated with Gould's Belt, the apparent disk of $\mathrm{OB}$ stars, gas, and dust in a great circle about the Sun inclined some $20^{\circ}$ to the Galactic plane (e.g., Stothers and Frogel 1974). The maximum extension to positive latitude of the Belt lies in the general direction 
of the Galactic center and is marked by the large clouds in Lupus, Ophiuchus, and Aquila; the Taurus and Orion clouds opposite in the sky mark the maximum negative-latitude extension of the Belt. The $\mathrm{CO}$ maps provide an important observational link between the optical dark clouds and associated stars of the Belt and the $21 \mathrm{~cm}$ "feature A" of Lindblad et al. (1973) (see $\S$ IV). As emphasized by Dame and Thaddeus (1985), the CO clouds are closely correlated spatially with the dark nebulae, while many are closely correlated in velocity with feature A.

Another possible large-scale symmetry, evident in both Figure 2 and Figure 3, is formed by regions of intense, complex emission toward Cygnus $X \quad\left(l \sim 80^{\circ}\right)$ and toward Vela $\left(l \sim 270^{\circ}\right)$, which, as Murphy $(1985)$ recently noted, lie in the Galactic plane $\sim 180^{\circ}$ apart and are fairly similar in overall intensity, angular size, velocity extent, and general structure. Further, these regions coincide with strong concentrations of Population I radio and optical tracers spread over a considerable range of distances. The CO data are evidently consistent with the idea long discussed (e.g., Bok 1959; Humphreys 1970; Walborn 1973; Herbst 1975; Vega, Muzzio, and Feinstein 1986) that the Sun is located in a Local spiral arm, seen tangentially toward Cygnus and Vela (see $§$ IV).

\section{LOCAL MOLECULAR CLOUDS}

The wide latitude coverage along the entire Milky Way of the composite survey allows the thickness and density of molecular gas in the solar neighborhood to be determined for the first time from a direct inventory of nearby molecular clouds. Previous CO estimates have been derived by extrapolating data from the inner and outer Galaxy to the solar circle (e.g., Sanders, Solomon, and Scoville 1984; Thaddeus and Chanan 1985) or have largely been based on local observations in only one quadrant (Dame and Thaddeus 1985).

Following Dame and Thaddeus (1985), we define local to mean within $1 \mathrm{kpc}$ of the Sun. Clouds this close lie in the low-velocity lane in the longitude-velocity map (Fig. 3) at absolute velocities less than $20 \mathrm{~km} \mathrm{~s}^{-1}$. In most directions such clouds can be readily distinguished from more distant ones by their low velocity and wide latitude extent; many are visible as dark nebulae on optical photographs. Distances, all taken from the literature, are based on associated Population I objects or on star counts or on the relation of visual absorption versus distance for stars within the cloud boundaries (e.g., Forbes 1985). Kinematic distances, although generally so inaccurate for such nearby objects as to be almost useless, were taken into account in a few cases (e.g., the " $-12 \mathrm{~km} / \mathrm{s}$ " clouds and Cepheus), but were never the sole or primary distance indicator. The change in $R_{\odot}$ from 10 to $8.5 \mathrm{kpc}$, recommended by IAU Commission 33 (Kerr and Lynden-Bell 1986), therefore has virtually no effect on the results presented below.

As in the analysis of nearly all the large-scale CO surveys, masses for the local clouds were computed by assuming a linear proportionality between $W_{\mathrm{CO}}$, the velocity-integrated $\mathrm{CO}$ intensity, and $\mathrm{N}_{\mathrm{H}_{2}}$, the $\mathrm{H}_{2}$ column density. We adopted the ratio $N_{\mathrm{H}_{2}} / W_{\mathrm{CO}}=2.7 \times 10^{20} \mathrm{~cm}^{-2} \mathrm{~K}^{-1} \mathrm{~km} \mathrm{~s}^{-1}$ derived by Bloeman et al. (1986) from intercomparison of $\mathrm{CO}, 21 \mathrm{~cm}$, and $\gamma$-ray surveys over large areas of the Galaxy (see Dame and Thaddeus 1985), since this determination agrees very closely with the results of a similar analysis of one of the most massive local molecular cloud complexes: the Orion region (Bloemen et al. 1984). Masses thus obtained are independent of uncertainties in the absolute calibration of the telescopes; they depend only on the calibration relative to the survey of Dame and Thaddeus (1985) used by Bloemen et al. (1986). Observations of reference $\mathrm{CO}$ sources along the Galactic plane confirm that the relative calibration is accurate to $5 \%$. To account for helium and heavier elements in the clouds, we assumed a mean molecular weight per $\mathrm{H}_{2}$ molecule of $2.76 \mathrm{~m}_{\mathrm{H}}$.

Individual local clouds or cloud complexes are listed in Table 2 with their limits in $l$ and $b$, mean velocities, distances, masses, mass-weighted mean displacement from the Galactic plane, $\bar{z}$, and rms dispersion about the plane, $\sigma_{z}$. Two sets of $l$ and $b$ limits are given for the Aquila Rift, Per OB2, and $\rho$ Oph because, owing to their irregular shapes, none of these could be enclosed by a single rectangular region without including other clouds or large, emission-free regions. Masses were determined directly from the $\mathrm{CO}$ emission within the rectangular regions integrated over the total velocity extents of the clouds.

The cloud boundaries given in Table 2 are in places somewhat arbitrary (e.g., between the Vul and Cygnus Rifts) and when so should be considered rough divisions between regions with nearly constant velocity and distance rather than as defining individual clouds. Neither the Lindblad Ring nor the " $-12 \mathrm{~km} / \mathrm{s}$ " emission is a single object; both appear to consist rather of fairly complex systems of clouds at nearly constant distance. Similarly, the limits given for any other region, such as the Aquila Rift or Cyg OB7, might contain smaller but presumably related clouds whose distances cannot be determined individually. The derived masses are typically $\sim 50 \%$ larger than in the first quadrant analysis of Dame and Thaddeus (1985) as a consequence of the larger cloud boundaries adopted here.

The complex, widespread emission in the second quadrant requires special attention. As mentioned (§ III), this emission is clearly divided between the Perseus arm near $-40 \mathrm{~km} \mathrm{~s}^{-1}$ and local material between -20 and $10 \mathrm{~km} \mathrm{~s}^{-1}$ (see Fig. 3). The local emission, however, appears further divided by a gap near $-7 \mathrm{~km} \mathrm{~s}^{-1}$. The more negative local component, centered near $-12 \mathrm{~km} \mathrm{~s}^{-1}$, contains several large clouds and smaller fragments plausibly related to a string of $O B$ associations-Cep OB2, Cep OB3, Cep OB4, and Per OB3-between $800 \mathrm{pc}$ and $1000 \mathrm{pc}$ from the Sun (Humphreys 1978). The Cep OB3 cloud has been studied in detail by Sargent $(1977,1979)$, and parts of other clouds in the region have been observed by Sargent et al. (1981), Elmegreen, Dickinson, and Lada (1978), and Casoli, Combes, and Gerin (1984); the distances derived or adopted, as well as the kinematic distances to most of the clouds, are in rough agreement with those of the OB associations. Further, the CN absorption-line study of Münch (1964) toward the H II region S171, lying toward the Cep OB4 molecular cloud, reveals strong $\mathrm{CN}$ absorption at a distance of $900 \mathrm{pc}$ (Osterbrock 1957). We therefore adopt $800 \mathrm{pc}$ as the distance to all the clouds in the more negative velocity local component and call them the " $-12 \mathrm{~km} / \mathrm{s}$ " clouds.

Two local velocity components are also seen in $21 \mathrm{~cm}$ observations over most of the Galactic plane (Lindblad 1974). The $21 \mathrm{~cm}$ component that corresponds to the " $-12 \mathrm{~km} / \mathrm{s}$ " clouds is identified with the Local spiral arm by Lindblad (1974), and the other, closer to $0 \mathrm{~km} \mathrm{~s}^{-1}$, is feature A, modeled by Lindblad et al. (1973) as an expanding shell of cold gas surrounding the Sun. In the second quadrant, Lindblad's "expanding ring" lies on average at $\sim 300 \mathrm{pc}$, in reasonable agreement with the distances to the nearest dust clouds in this direction (Elmegreen 1982). The low velocity of the CO emission and the very large latitude extent of the $21 \mathrm{~cm}$ feature A suggest that the material is close-significantly closer than the " -12 
TABLE 2

Molecular Gas Within 1 Kiloparsec OF SUN

\begin{tabular}{|c|c|c|c|c|c|c|c|c|c|c|}
\hline Region & $l_{\min }$ & $l_{\max }$ & $b_{\min }$ & $b_{\max }$ & $\left(\begin{array}{c}v \\
\left(\mathrm{~km} \mathrm{~s}^{-1}\right)\end{array}\right.$ & $\begin{array}{c}D \\
(\mathrm{pc})\end{array}$ & Ref. & $\begin{array}{c}M \\
\left(10^{5} M_{\odot}\right)\end{array}$ & $\begin{array}{c}\bar{z}^{\mathrm{a}} \\
(\mathrm{pc})\end{array}$ & $\begin{array}{l}\sigma_{z}^{b} \\
(\mathrm{pc})\end{array}$ \\
\hline Aquila Rift $\ldots \ldots \ldots \ldots \ldots$ & $\begin{array}{l}18.5 \\
34\end{array}$ & $\begin{array}{l}34^{\circ} \\
44^{\circ}\end{array}$ & $\begin{array}{l}-6^{\circ} \\
-4\end{array}$ & $\left.\begin{array}{c}10^{\circ} \\
4\end{array}\right\}$ & 8 & 200 & 1 & 1.5 & 9 & 15 \\
\hline Cloud A $\ldots \ldots \ldots \ldots \ldots$ & 44 & 49.5 & -4 & 2 & 27 & 500 & 1 & $0.4^{\mathrm{c}}$ & -7 & 14 \\
\hline Cloud B $\ldots \ldots \ldots \ldots \ldots$. & 44 & 54 & -4 & 5 & 7 & 300 & 1 & $0.4^{\mathrm{c}}$ & 0 & 11 \\
\hline Cloud $\mathrm{C}$................. & 50 & 55 & -1 & 3.5 & 24 & 500 & 1 & 0.3 & 5 & 12 \\
\hline Vul Rift $\ldots \ldots \ldots \ldots \ldots$ & 54 & 63 & -3 & 5 & 10 & 400 & 1 & 0.8 & 5 & 12 \\
\hline Cyg Rift $\ldots \ldots \ldots \ldots \ldots$ & 63 & 86.5 & -4 & 4 & 7 & 700 & 1 & $8.6^{\mathrm{d}}$ & -4 & 24 \\
\hline Cyg OB7 $\ldots \ldots \ldots \ldots \ldots$ & 87 & 99 & -3 & 8 & -1 & 800 & 1 & 7.5 & 41 & 52 \\
\hline Lindblad Ring .......... & 100 & 164 & -4 & 10 & 1 & 300 & 2 & 1.6 & 22 & 28 \\
\hline$"-12 \mathrm{~km} / \mathrm{s} " \ldots \ldots \ldots \ldots$ & 102 & 161 & -4 & 10 & -12 & 800 & 3 & 8.7 & 44 & 59 \\
\hline Cepheus $\ldots . \ldots \ldots \ldots \ldots$. & 100 & 120 & 11 & 22 & -5 & 450 & 4 & 1.9 & 131 & 133 \\
\hline Taurus $\ldots \ldots \ldots \ldots \ldots \ldots$ & 163 & 178 & -22 & -9.5 & 5 & 140 & 5 & 0.3 & -37 & 38 \\
\hline Per OB2 $\ldots \ldots \ldots \ldots \ldots$ & $\left\{\begin{array}{l}154 \\
163\end{array}\right.$ & $\begin{array}{l}162.5 \\
171\end{array}$ & $\begin{array}{r}-25 \\
-9\end{array}$ & $\begin{array}{l}-7 \\
-6\end{array}$ & $\left.\begin{array}{r}5 \\
-3\end{array}\right\}$ & 350 & 5 & 1.3 & -84 & 92 \\
\hline Mon OB1. & 197.5 & 205 & -1 & 4 & 7 & 800 & 7 & 1.6 & 17 & 22 \\
\hline Orion $A \quad \ldots \ldots \ldots \ldots \ldots$. & 208.5 & 218 & -21 & -14.5 & 5 & 500 & 6 & 1.6 & -163 & 164 \\
\hline Orion B $\ldots \ldots \ldots \ldots \ldots \ldots$. & 202.5 & 208 & -21 & -6 & 5 & 500 & 6 & 1.7 & -129 & 132 \\
\hline Mon R2 ............... & 210 & 218 & -14 & -10 & 7 & 830 & 6 & 1.2 & -182 & 183 \\
\hline Vela Sheet $\ldots \ldots \ldots \ldots \ldots$ & 272 & 279 & -3 & 8 & 0 & 425 & 8 & 0.8 & 9 & 19 \\
\hline Cham $\ldots \ldots \ldots \ldots \ldots \ldots$ & 295 & 305 & -20 & -12 & 4 & 215 & 9 & 0.1 & -60 & 60 \\
\hline Coalsack $\ldots \ldots \ldots \ldots \ldots \ldots$ & 300 & 307 & -4 & 3 & -4 & 175 & 10 & 0.04 & -2 & 5 \\
\hline$G 317-4 \ldots \ldots \ldots \ldots \ldots$ & 315 & 320 & -6 & -2 & -6 & 170 & 11 & 0.03 & -11 & 11 \\
\hline Lupus $\ldots \ldots \ldots \ldots \ldots \ldots \ldots$ & 333 & 346 & 4 & 22 & 5 & 170 & 8 & 0.3 & 32 & 35 \\
\hline$\rho$ Oph $\ldots \ldots \ldots \ldots \ldots \ldots \ldots$ & $\left\{\begin{array}{l}350 \\
356\end{array}\right.$ & $\begin{array}{l}2 \\
5\end{array}$ & $\begin{array}{r}13 \\
3\end{array}$ & $\left.\begin{array}{l}24 \\
12.5\end{array}\right\}$ & 3 & 165 & 12 & 0.3 & 35 & 39 \\
\hline R CrA & 357 & 4 & -22 & -14 & 6 & 150 & 13 & 0.03 & -55 & 55 \\
\hline
\end{tabular}

a $\bar{z}=\Sigma m_{i} z_{i} / \Sigma m_{i} ;$ using bins 10 pc wide in $z$.

b $\sigma_{z}=\left[\Sigma m_{i} z_{i}^{2} / \Sigma m_{i}\right]^{1 / 2}$; using bins $10 \mathrm{pc}$ wide in $z$.

c The boundaries of this cloud are 2-3 times larger than the ones adopted by Dame and Thaddeus (1985) and include adjacent, presumably related gas.

${ }^{d}$ Following Dame and Thaddeus (1985), the average CO intensity of the Cygnus Rift above $l=74^{\circ}$, where the Rift is confused with the more distant and intense Cyg X region, was assumed to be the same as that measured below $74^{\circ}$, where only the Rift is seen.

DisTANCE REFERENCES. - (1) Dame and Thaddeus 1985; (2) Lindblad et al. 1973; (3) Humphreys 1978; (4) Lebrun 1986; (5) Ungerechts and Thaddeus 1987; (6) Maddelena et al. 1986; (7) Blitz 1978; (8) Murphy 1985; (9) Hyland, Jones, and Mitchell 1982; (10) Rodger 1960;(11) Neckel and Klare 1980;(12) Chini 1981;(13) Gaposchkin and Greenstein 1936.

$\mathrm{km} / \mathrm{s}$ " clouds. We therefore adopt a distance of $300 \mathrm{pc}$ for the CO clouds coincident in velocity with the $21 \mathrm{~cm}$ feature A and designate them the Lindblad Ring clouds.

The local molecular clouds are shown in projection on the Galactic plane in Figure 7. The distribution is far from random: nearly all the clouds in the first and fourth quadrants apparently lie on a fairly straight ridge more than $1 \mathrm{kpc}$ long, running from the Vela Sheet to the Cygnus Rift. The clouds from Chamaeleon to the Vul Rift may be related to Lindblad's expanding ring, although the fourth quadrant clouds are much farther from the Sun than in the model of Lindblad et al. (1973). The entire ridge may trace the inner edge of the Local spiral arm, and it is possible that the large Vela Sheet and Cyg Rift clouds at either end are superpositions of clouds which lie even farther along the ridge. The overall distribution of clouds is consistent with the Sun lying near the inner edge of the Local spiral arm; the large apparently empty region in the first and fourth quadrants below the ridge is the interarm region between the Local arm and the Sagittarius arm, which at $l=0^{\circ}$ lies $\sim 1700 \mathrm{pc}$ from the Sun (Dame et al. 1986). The existence of this large interarm void in molecular clouds and presumably dark nebulae explains why in directions such as that toward M17 at $l=14^{\circ}$, where there happens to be little local obscuration in the Rift system, it is readily possible to observe optical objects in the Sagittarius arm.

The mean density of molecular gas within $1 \mathrm{kpc}$ of the Sun
(Table 2) is shown in Figure 8 as a function of distance from the Galactic plane $z$, broken down into the contribution from each Galactic quadrant. This figure is an extension to all four quadrants of Figure 11 of Dame and Thaddeus (1985), with the trivial difference that differential mass per parsec in $z$ has been converted to density. The disproportionate amount of molecular gas in the northern Milky Way is evident: in the first and second quadrants the molecular mass is 4 times greater than in the third and fourth.

Since about half the local molecular mass is contained in five fairly compact objects (Cyg Rift, Cyg OB7, Cepheus, Orion B, and Mon OB1), small number fluctuations are significant, and within $1 \mathrm{kpc}$ the average shape of the Galactic distribution in $z$ at the solar circle is not well sampled and therefore not determined very accurately. Generally, a Gaussian distribution in $z$ has been adopted in the analysis of the large-scale distribution of Clouds in the Galaxy, and Grabelsky et al. (1987) showed that a Gaussian is indeed a good approximation to the $z$ distribution of clouds in the Carina arm. Simply fitting a Gaussian to the clouds in Table 2, however, because of the poor sampling makes little sense. Although this small sample of clouds does not allow determination of all moments of the $z$ distribution, the first two moments can be determined: the mean $z$ displacement of the distribution is $+5 \mathrm{pc}$ and its $\mathrm{rms}$ dispersion about the plane is $74 \mathrm{pc}$. Given that from other evidence molecular clouds appear to follow a Gaussian $z$ dis- 


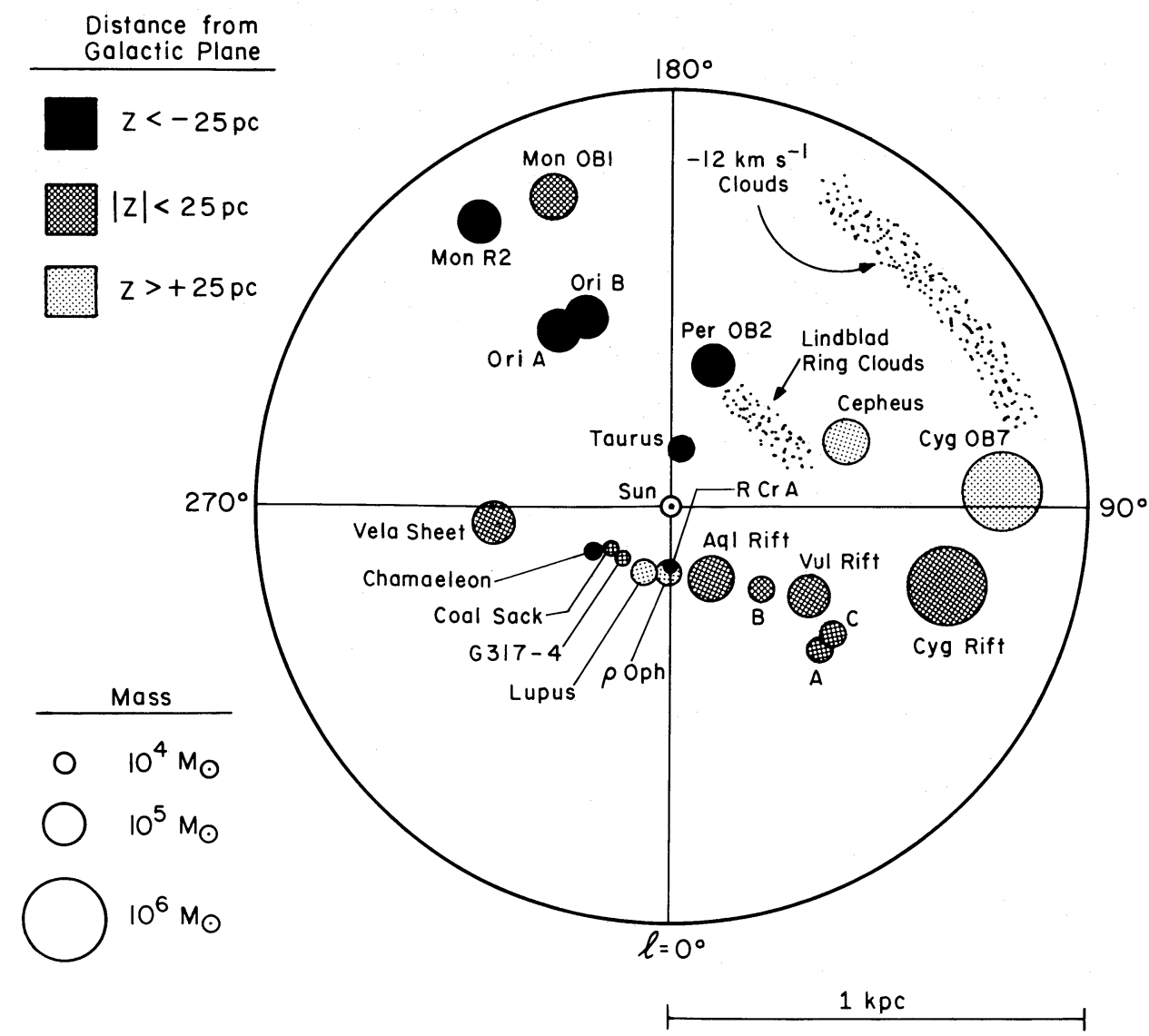

FIG. 7.-The distribution in the Galactic plane of molecular clouds within $1 \mathrm{kpc}$ of the Sun (Table 2). The circle radii are proportional to the cube roots of the cloud masses and in most cases are close to the clouds' actual radii. The shading indicates distance from the Galactic plane.

The general regions of the " $-12 \mathrm{~km} / \mathrm{s}$ " and Lindblad Ring clouds are indicated but individual clouds are not shown. The widths of these regions in heliocentric distance are unknown; the widths shown are arbitrary.

tribution, our best estimate of the molecular layer halfthickness at the solar circle is $87 \mathrm{pc}$, the half-thickness at half-intensity of a Gaussian with a dispersion of $74 \mathrm{pc}$.

Similarly, our best estimate of the mean midplane density at the solar circle is obtained by assuming that, on average, molecular gas has a Gaussian distribution in $z$. The total mass of molecular clouds within $1 \mathrm{kpc}$ of the Sun is $4.0 \times 10^{6} \mathrm{M}_{\odot}$, implying a mean surface density of $1.3 \mathrm{M}_{\odot} \mathrm{pc}^{-2}$. If a Gaussian distribution in $z$ with a half-thickness of $87 \mathrm{pc}$ is then assumed, the mean midplane density is $0.0068 \mathrm{M}_{\odot} \mathrm{pc}^{-3}$, or $0.10 \mathrm{H}_{2}$ $\mathrm{cm}^{-3}$. The value at $z=0 \mathrm{pc}$ in Figure 8 is about twice as high, presumably simply because of statistical fluctuations in the $z$ distribution of clouds.
Table 3 summarizes the local scale height and density of molecular gas and compares our results to the first quadrant results of Dame and Thaddeus (1985). As expected, the surface density averaged over all four quadrants is lower than that averaged over only the first, as that contains the large clouds of the Great Rift. The increased layer thickness also is not surprising: Dame and Thaddeus based their results on the dispersion of only the first quadrant and Orion clouds; the present larger survey includes other high $z$ clouds, in particular those in Taurus and Cepheus. With a smaller total mass spread over a larger $z$ range, the average midplane density derived here is almost a factor of 2 below the value derived from the first quadrant alone.

TABLE 3

MoleCUlar Gas Within 1 KiLOPARSEC

\begin{tabular}{|c|c|c|}
\hline & $\begin{array}{c}l=12^{\circ}-100^{\circ} \text { only: } \\
\text { Dame and Thaddeus }(1985)\end{array}$ & $\begin{array}{l}\text { All quadrants: } \\
\text { Present work }\end{array}$ \\
\hline rms $z$ dispersion $\ldots \ldots \ldots \ldots$ & $64 \mathrm{pc}$ & $74 \mathrm{pc}$ \\
\hline Layer thickness (HWHM) & $75 \mathrm{pc}$ & $87 \mathrm{pc}$ \\
\hline Mass $\ldots \ldots \ldots \ldots \ldots \ldots \ldots$ & $6.4 \times 10^{6} M_{\odot}^{\mathrm{a}}$ & $4.0 \times 10^{6} M_{\odot}$ \\
\hline Surface density $\ldots \ldots \ldots \ldots \ldots \ldots \ldots$ & $2.0 M_{\odot} \mathrm{pc}^{-2}$ & $1.3 M_{\odot} \mathrm{pc}^{-2}$ \\
\hline \multirow[t]{2}{*}{ Midplane density .................... } & $0.013 M_{\odot} \mathrm{pc}^{-3}$ & $0.0068 M_{\odot} \mathrm{pc}^{-3}$ \\
\hline & $0.20 \mathrm{H}_{2} \mathrm{~cm}^{-3}$ & $0.10 \mathrm{H}_{2} \mathrm{~cm}^{-3}$ \\
\hline
\end{tabular}

${ }^{\mathrm{a}}$ Four times the mass in the quadrant observed $\left(l=12^{\circ}-100^{\circ}\right)$. 


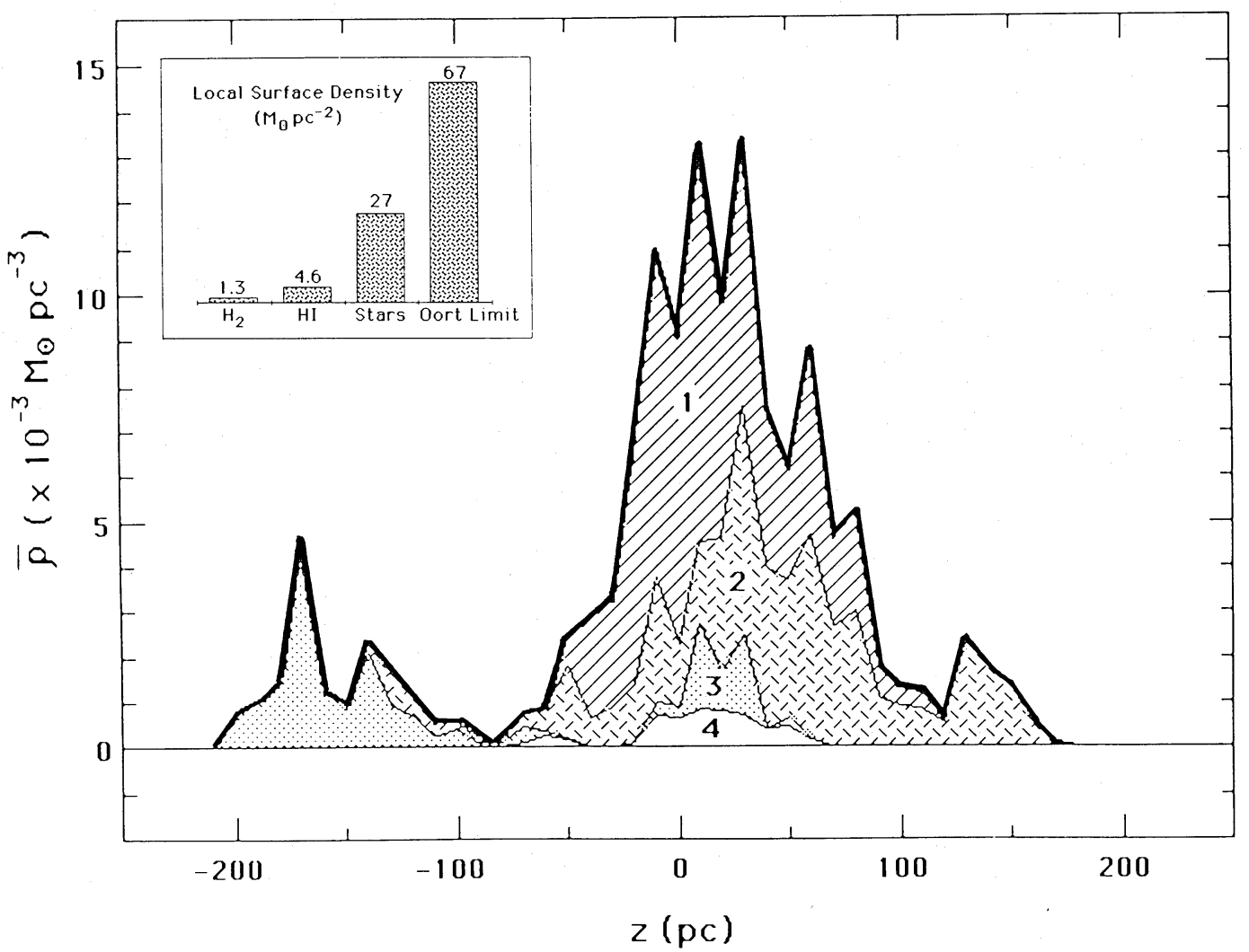

FIG. 8.-The mean density of molecular gas within $1 \mathrm{kpc}$ of the Sun as a function of distance from the Galactic plane $z$ (heavy upper line). Integrated CO intensity, $W_{\mathrm{CO}}$, was converted to mass (see $\S \mathrm{IV}$ ) and added into bins $10 \mathrm{pc}$ wide in $z$. Shading indicates the contributions from each of the four Galactic quadrants, 1 from $l=00^{\circ}$ to $90^{\circ}, 2$ from $l=90^{\circ}$ to $180^{\circ}$, etc. Insert: mass surface densities in the solar neighborhood; $\mathrm{H}_{2}$ from the present work; $\mathrm{H}$ I from Burton (1976), assuming a mean weight per $\mathrm{H}_{\mathrm{I}}$ atom of $1.38 \mathrm{~m}_{\mathrm{H}}$; stars from Miller and Scalo (1979); and the Oort limit from Bahcall (1984).

Our direct measurements of the local molecular scale height and density agree well with results from the inner and outer Galaxy extrapolated to the solar circle. The inner Galaxy axisymmetric analyses of Bronfman et al. (1987) and Sanders, Solomon, and Scoville (1984) yield a half-thickness at the solar circle of $\sim 75 \mathrm{pc}$, while the analysis of Carina arm clouds in the outer Galaxy by Grabelsky et al. (1987) yields $\sim 100 \mathrm{pc}$ at the solar circle, nicely bracketing the present $87 \mathrm{pc}$. Extrapolations of the densities of Bronfman et al. (1987) and Grabelsky et al. (1987) to the solar circle yield midplane densities of $\sim 0.01 M_{\odot}$ $\mathrm{pc}^{-3}$, in rough agreement with our value of $0.0068 \mathrm{M}_{\odot} \mathrm{pc}^{-3}$. Although the value derived by Sanders, Solomon, and Scoville (1984) is more than 4 times higher, this difference can be attributed in part to differences in instrumental calibration and adopted $N_{\mathrm{H}_{2}} / W_{\mathrm{CO}}$ ratios; Bronfman et al. (1987) show that the remaining discrepancy is mainly due to a difference in fitting procedures.

The agreement of our measurement of the local density with extrapolations of azimuthal averages within and beyond the solar circle implies that, averaged over a circular area of $1 \mathrm{kpc}$ radius, the molecular density near the Sun is typical of that at the solar circle. Within this area, however, a large-scale asymmetry exists, the density in the northern half $\left(l=0^{\circ}\right.$ to $\left.180^{\circ}\right)$ being 4 times larger than that in the southern half $\left(180^{\circ}\right.$ to $360^{\circ}$ ).

The relative contribution of $\mathrm{H}_{2}$ to the total local interstellar mass is evident from the insert to Figure 8 . The surface density of $\mathrm{H}_{2}$ is less than a third that of $\mathrm{H} \mathrm{I}$, and the total mass of gas $\left(\mathrm{H}_{2}+\mathrm{H}_{\mathrm{I}}\right)$ is about a fifth of the total mass in stars. In turn, the total mass in gas and stars is only about half of the Oort limit, the total mass in the solar neighborhood deduced from the observed distribution of stars perpendicular to the Galactic plane. The present work is conclusive evidence that the "missing mass" in the solar neighborhood, the difference between the total mass observed and the Oort limit, is not in the form of molecular clouds.

Finally, it is instructive to compare our results with those from a completely independent study of the local $\mathrm{H}_{2}$ gas by Savage et al. (1977), who used Copernicus UV absorption spectra of the Lyman bands toward early-type stars to determine $\mathrm{H}_{2}$ column densities. The 109 stars they analyzed are distributed fairly uniformly in Galactic longitude and about $85 \%$ fall within $25^{\circ}$ of the plane; however, as they point out, their survey is biased toward stars with less than normal reddening per unit distance; all but 18 lie outside the lowest contour in Figure 2 and some may lie in front of our $\mathrm{CO}$ clouds. Because of this bias, the mean $\mathrm{H}_{2}$ density along lines of sights to these stars, $0.036 \mathrm{~cm}^{-3}$, is not a useful measure of the total $\mathrm{H}_{2}$ density locally; it is valuable, however, as an upper limit to the amount of $\mathrm{H}_{2}$ that lies outside the lowest contour in Figure 2, possibly in the low-density halos of clouds or in clouds deficient in CO. Our midplane density might, at most, be corrected upward by one-third to account for such gas.

In an attempt to remove their bias toward directions of low $\mathrm{H}_{2}$ column density, Savage et al. used the large-scale extinction measurements of FitzGerald (1968) to extrapolate to higher density regions. They derived a mean $\mathrm{H}_{2}$ density in the Galactic plane within $500 \mathrm{pc}$ of the Sun of $0.14 \mathrm{~cm}^{-3}$. In comparison, 
from Table 2 the average midplane density out to $500 \mathrm{pc}$ (again with $87 \mathrm{pc}$ as the layer half-thickness) is found to be $0.13 \mathrm{~cm}^{-3}$. This close agreement is undoubtedly fortuitous, but taken loosely it implies that most of the matter in the local clouds is not highly opaque at visual wavelengths. It is evidence too for the general paradigm that nearly all dark nebulae are molecular clouds and vice versa.

\section{COMPLETENESS}

Since many of the large local clouds lie more than $10^{\circ}$ from the Galactic plane, beyond the latitude range of the composite survey at some longitudes, it is appropriate to ask whether similar clouds might exist in unobserved regions (see the sampling boundary in Fig. 2). Although many small clouds undoubtedly exist beyond the composite survey, such as those associated with the IRAS high-latitude "cirrus" (Magnani, Blitz, and Mundy 1985; de Vries, Heithausen, and Thaddeus 1987), comparison of our CO data with other Population I surveys indicates that no large clouds have escaped detection.

Figure 9 presents a comparison of four large-scale tracers of interstellar gas. With the exception of the CO map, each is complete out to at least $\pm 20^{\circ}$ of Galactic latitude. The darkcloud map was adapted from a map with $\sim 4^{\circ}$ angular resolution produced by Feitzinger and Stüwe (1986b), while the angular resolution of the $\operatorname{COS} B$ gamma-ray data is greater than $2^{\circ}$; the $\mathrm{CO}$ and infrared maps are smoothed to 2.5 angular resolution for comparison. The three contours in each map were chosen to emphasize the similarity of the maps.

Some of the obvious differences among the maps are easily understood. The dark-cloud map lacks the intense innerGalaxy ridge, because only relatively nearby molecular clouds are observed as dark clouds. The $\mathrm{CO}$ emission is more narrowly confined in latitude because it traces the distribution of only molecular gas; the other maps also trace atomic gas, which has a scale height in $z$ about twice as large as molecular gas. Further, the gamma-ray map contains several point sources, notably the Vela pulsar near $l=263^{\circ}$ and the Crab pulsar near $l=184^{\circ}$.

Allowing for these differences, the similarity in the distribution of all the Population I tracers is striking. The $\mathrm{CO}$ and dark-cloud maps show the most detailed correlation, with an almost one-to-one correspondence of clouds; even the small
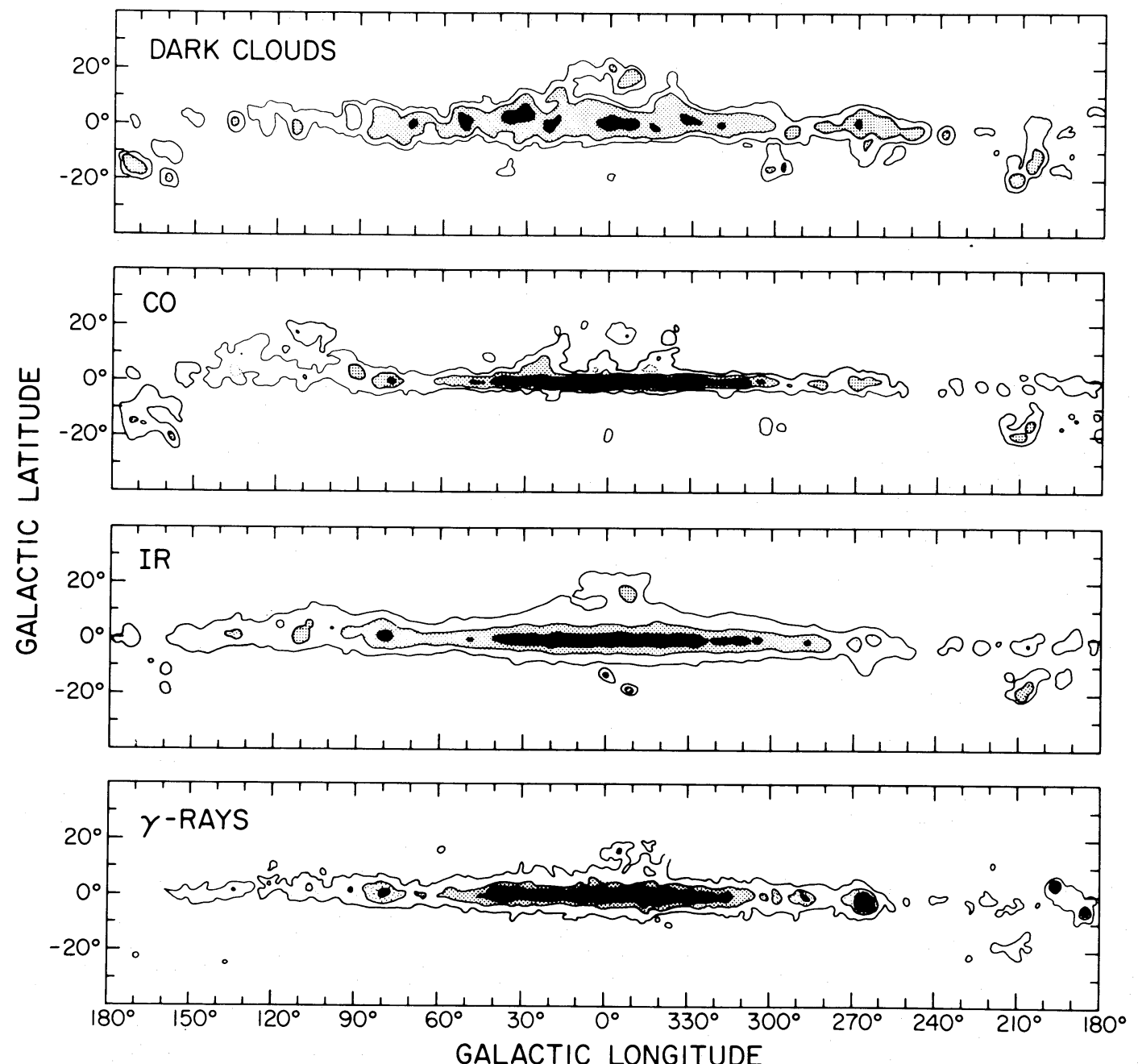

FIG. 9.-Dark clouds: The Galactic distribution of 2622 dark clouds folded with a Gaussian point-spread function of the form $\exp \left(-\theta / \theta_{0}\right)$, with $\theta_{0}=2.5$. The contours are at opacity classes 1, 2, and 5; adapted from Fig. 1 of Feitzinger and Stüwe (1986b). CO: Velocity-integrated CO emission from the Milky Way (Fig. 2) smoothed to an angular resolution of 2.5 (FWHM). Contours are at 2,9 , and $23 \mathrm{~K} \mathrm{~km} \mathrm{~s}^{-1}$. IR: IRAS all-sky map at $100 \mu \mathrm{m}$ with zodiacal emission removed (Boulanger 1987), smoothed to an angular resolution of 2.5 (FWHM). Contours are at 39, 90, and $500 \mathrm{MJy} \mathrm{sr}^{-1}$. Gamma rays: Galactic gamma-ray emission in the energy range $70 \mathrm{MeV}-5 \mathrm{GeV}$, observed by the $\operatorname{COS} B$ satellite. Contours are at 6,12 , and 18 on-axis counts s ${ }^{-1} \mathrm{sr}^{-1}$. Adapted from Fig. 4 of Mayer-Hasselwander $e t$ al. (1982). 
dark cloud near $l=35^{\circ}$ was detected in CO (Lebrun 1986), although its emission lies below the lowest contour in Figure 9. The absence of the Cepheus cloud in the dark-cloud map is somewhat puzzling, but a general flaring of the dark clouds near $l=110^{\circ}$ is clearly seen in the unsmoothed data (Feitzinger and Stüwe 1986b).

A detailed comparison of the $\mathrm{CO}$ data and both the infrared and gamma-ray data is beyond the scope of this paper, but, like the dark-cloud map, the infrared and gamma-ray maps in Figure 9 offer little evidence for large objects outside areas observed in $\mathrm{CO}$. One exception may be the region above the plane near $l=0^{\circ}$ : the other gas tracers suggest that low-level emission unobserved in $\mathrm{CO}$ (Fig. 2) may connect the Aquila Rift, $\rho$ Oph, and Lupus clouds. Further CO observations are planned to fill these possibly significant holes in our composite survey.

\section{SUMMARY}

Large-scale $\mathrm{CO}$ surveys of the entire Galactic plane and specific nearby clouds have been combined to produce a panorama of the entire Milky Way in molecular clouds at an angular resolution of $\frac{1}{2}^{\circ}$. Covering $10^{\circ}-20^{\circ}$ in latitude at all longitudes and all or nearly all large, nearby clouds at higher latitude, the composite survey is the only molecular line survey to date with sky coverage and resolution comparable to that of the early $21 \mathrm{~cm}$ surveys.

The inner Galaxy spiral arms produce, as expected, a thin, intense ridge of emission along the Galactic plane within $\sim 60^{\circ}$ of the Galactic center. The local emission, covering a much wider latitude range at low velocity, shows the same large-scale features as the distribution of dark clouds: the large Lupus, $\rho$ Oph, and Aquila Rift clouds lie at the positive-latitude extension of Gould's Belt, while opposite in the sky the Orion and Taurus clouds mark the negative-latitude extension. The much higher density of dark clouds in the northern Milky Way versus the southern, obvious on large-scale photographs, is evident also in CO. Within $1 \mathrm{kpc}$ of the Sun, the molecular mass in the first and second quadrants is 4 times greater than in the third and fourth.

The complete longitude and wide latitude coverage of the survey provides a thorough inventory of large molecular clouds near the Sun. The overall distribution of clouds within 1 $\mathrm{kpc}$ is consistent with the Sun lying near the inner edge of a local spiral arm or spur. The half-thickness at half-intensity of the local molecular cloud layer is $87 \mathrm{pc}$, in good agreement with results from the inner and outer Galaxy, and the mean midplane density, derived assuming a Gaussian $z$ distribution, is $\sim 0.0068 \mathrm{M}_{\odot} \mathrm{pc}^{-3}$.

We thank D. Grabelsky, Y.-L. Huang, M. Koprucu, F. Lebrun, and R. J. Maddalena for providing data, some in advance of publication; F. Avilés, H. Alvarez, L. Bronfman, J. Montani, and E. S. Palmer for help in operating and maintaining the New York and Chile telescopes; A. V. Smith for help with data analysis; and E. Sarot for editorial assistance. L.- $\AA$. N. acknowledges support from the Swedish Natural Science Research Council and H. U. acknowledges support from the Alexander von Humboldt Foundation through a Fedor-Lynen Fellowship.
Bahcall, J. N. 1984, Ap. J., 276, 169

Bitran, M., Thaddeus, P., and Cohen, R. S. 1987, in preparation.

Blitz, L. 1978, Ph.D. thesis, Columbia University.

Blitz, L., and Thaddeus, P. 1980, Ap. J., 241, 676.

Bloemen, J. B. G. M., Caraveo, P. A., Hermsen, W., Lebrun, F., Maddalena,

R. J., Strong, A. W., and Thaddeus, P. 1984, Astr. Ap., 139, 37

Bloemen, J. B. G. M., et al. 1986, Astr. Ap., 154, 25.

Boulanger, F. 1987, private communication.

Bok, B. J. 1959, Observatory, 79, 58.

Bronfman, L. 1980, M.S. thesis, University of Chile.

Bronfman, L., Cohen, R. S., Alvarez, H., May, J., and Thaddeus, P. 1987, Ap. J., in press.

Burstein, D., and Heiles, C. 1978, Ap. J., 225, 40

Burton, W. B. 1976, Ann. Rev. Astr. Ap., 14, 275

Casoli, F., Combes, F., and Gerin, M. 1984, Astr. Ap., 133, 99.

Chini, R. 1981, Astr. Ap., 99, 346.

Christiansen, W. N., and Hindman, J. V. 1952, Australian J. Sci. Res., 5, 437.

Cohen, R. S. 1983, in Surveys of the Southern Galaxy, ed. W. B. Burton and F. P. Israel (Dordrecht: Reidel), 265.

Cohen, R. S., Cong, H., Dame, T. M., and Thaddeus, P. 1980, Ap. J. (Letters), 239, L53.

Cohen, R. S., Dame, T. M., and Thaddeus, P. 1986, Ap. J. Suppl., 60, 695

Cohen, R. S., Grabelsky, D. A., May, J., Bronfman, L., Alvarez, H. and Thad-

deus, P. 1985, Ap. J.(Letters), 290, L15.

Cohen, R. S. and Thaddeus, P. 1977, Ap. J.(Letters), 217, L155.

Cong, H.-I. Kerr, A. R., and Mattauch, R. J. 1979, IEEE Trans., MTT-27, 245.

Dame, T. M., and Thaddeus, P. 1985, Ap. J., 297, 751.

de Geus, E. J. 1987, private communication.

de Vries, H. W., Heithausen, A., and Thaddeus, P. 1987, Ap. J., 319, 723

Eggen, O. J. 1980, Ap. J., 238, 627.

Elmegreen, B. G. 1982, in Submillimeter Wave Astronomy, ed. J. E. Beckman

and J. P. Phillips (Cambridge: Cambridge University Press), p. 1.

Elmegreen, B. G., Dickinson, D. F., and Lada, C. J. 1978, Ap. J., 220, 853.

Ewen, H. I., and Purcell, E. M. 1951, Nature, 168, 356.

Feitzinger, J. V., and Stüwe, J. A. 1986a, Ap. J., 305, 534.

1986 b Vistas Astr, $29,291$.

FitzGerald, M. P. 1968, A.J., 73, 983

Forbes, D. 1985, A.J., 90, 301.

Gaposchkin, S., and Greenstein, J. L. 1936, Harvard Obs. Bull., No. 904.

FitzGerald, M. P. 1968, A.J., 73, 983.

Forbes, D. 1985, A.J., 90, 301.

Gaposchkin, S., and Greenstein, J. L. 1936, Harvard Obs. Bull., No. 904.

Gordon, M. A., and Burton, W. B. 1976, Ap.J., 208, 346.

\section{REFERENCES}

Grabelsky, D. A. 1985, Ph.D. thesis, Columbia University.

Grabelsky, D. A., Cohen, R. S., Bronfman, L., Thaddeus, P., and May, J. 1987, Ap. J., 315, 122

Grenier, I. A., Dame, T. M., and Thaddeus, P. 1987, private communication.

Heiles, C. 1976, Ap. J., 204, 379.

Herbst, W. 1975, A.J., 80, 503.

Huang, Y.-L. 1985, Ph.D. thesis, Columbia University.

Humphreys, R. M. 1970, A.J., 75, 602.

1978, Ap. J.Suppl., 38, 309.

Hyland, A. R., Jones, T. J., and Mitchell, R. M. 1982, M.N.R.A.S., 201, 1095

Keto, E. R., and Myers, P. C. 1986, Ap. J., 304, 466.

Kerr, F. J., Hindman, J. V., and Gum, C. S. 1959, Australian J. Phys., 12, 270.

Kerr, F. J, and Lynden-Bell, D. 1986, M.N.R.A.S., 221, 1023.

Koprucu, M., Cohen, R. S., and Thaddeus, P. 1987, private communication

Kutner, M. L., and Mead, K. N. 1985, in IAU Symposium 106, The Milky Way Galaxy, ed. H. van Woerden, R. J. Allen, and W. B. Burton (Dordrecht: Reidel), p. 209.

Lebrun, F. 1986, Ap. J., 306, 16

Lebrun, F., and Huang, Y.-L. 1984, Ap. J., 281, 634.

Lebrun, F., and Paul, J. A. 1983, Ap. J., 266, 276.

Lindblad, P. O. 1974, Highlights of Astronomy, 3, ed. G. Contopoulos, 381.

Lindblad, P. O., Grape, K., Sandqvist, Aa., and Schober, J. 1973, Astr. Ap., 24 309.

Loren, R. B., Peters, W. L., and Vanden Bout, P. A. 1974, Ap. J. (Letters), 194, L103.

Maddalena, R. J., Morris, M., Moscowitz, J., and Thaddeus, P. 1986, Ap. J., 303, 375.

Maddalena, R. J., and Thaddeus, P. 1985, Ap. J., 294, 231

Magnani, L., Blitz, L., and Mundy, L. 1985, Ap. J., 295, 402.

Mauzy, B. 1974, NRAO Electronics Division Internal Rept., No. 146.

May, J., Murphy, D. C., and Thaddeus, P. 1987, Astr. Ap. Suppl., in press.

Mayer-Hasselwander, H. A., et al. 1982, Astr. Ap., 105, 164

Miller, G. E., and Scalo, J. M. 1979, Ap. J. Suppl., 41, 513.

Muller, C. A., and Westerhout, G. 1957, Bull. Astr. Inst. Netherlands, 13, 151.

Münch, G. 1964, Ap. J., 140, 107.

Murphy, D. C. 1985, Ph.D. thesis, Massachusetts Institute of Technology.

Murphy, D. C. Cohen, R., and May, J. 1986, Astr. Ap., 167, 234.

Neckel, T., and Klare, G., 1980, Astr. Ap. Suppl., 42, 251.

Nyman, L.-Å., Alvarez, H., Cohen, R. S., and Thaddeus, P. 1987a, in preparation.

Nyman, L.-Å., Thaddeus, P., Bronfman, L., and Cohen, R. S. 1987b, Ap. J., 314 374

Osterbrock, D. E. 1957, Ap. J., 125, 622 
Pan, S.-K. 1984, Ph.D. thesis, Columbia University.

Penzias, A. A., and Burrus, C. A. 1973, Ann. Rev. Astr. Ap., 11, 51.

Rodgers, A. W. 1960, M.N.R.A.S., 120, 163.

Sanders, D. B., Solomon, P. M., and Scoville, N. Z. 1984, Ap. J., 276, 182.

Iol Sargent, A. I. 1977, Ap. J., 218, 736.

ol 1 1979, Ap.J., 233,163.

II Sargent, A. I., van Duinen, R. J., Nordh, H. L., and Aalders, J. W. G. 1981, Astr. Ap., 94, 377 .

Savage, B. D., Bohlin, R. C., Drake, J. F., and Budich, W. 1977, Ap. J., 216, 291.

Schlosser, W., and Görnandt, V. 1984, Astr. Ap., 137, 287.

R. S. CoHEN: 51 Seventh Ave. Apt 1, Brooklyn, NY 11217

T. M. Dame, L.-Å Nyman, and P. Thaddeus: Harvard-Smithsonian Center for Astrophysics, 60 Garden Street, Cambridge, MA 02138

E. J. DE Geus: Sterrewacht Leiden, P.O. Box 9513, 2300 RA Leiden, Netherlands

I. A. GrenIER: CEN-Saclay, DphG/SAP 91191, Gig-sur-Yvette, Cedex, France

J. MAY: Departamento de Astronomía, Universidad de Chile, Casilla 36-D, Santiago, Chile

D. C. MuRPhy: European Southern Observatory, Casilla 19001, Santiago, Chile

H. UngeReChTS: I. Physikalisches Institut der Universität zu Köln, Zülpicher Strasse 77, 5000 Köln 41, West Germany 

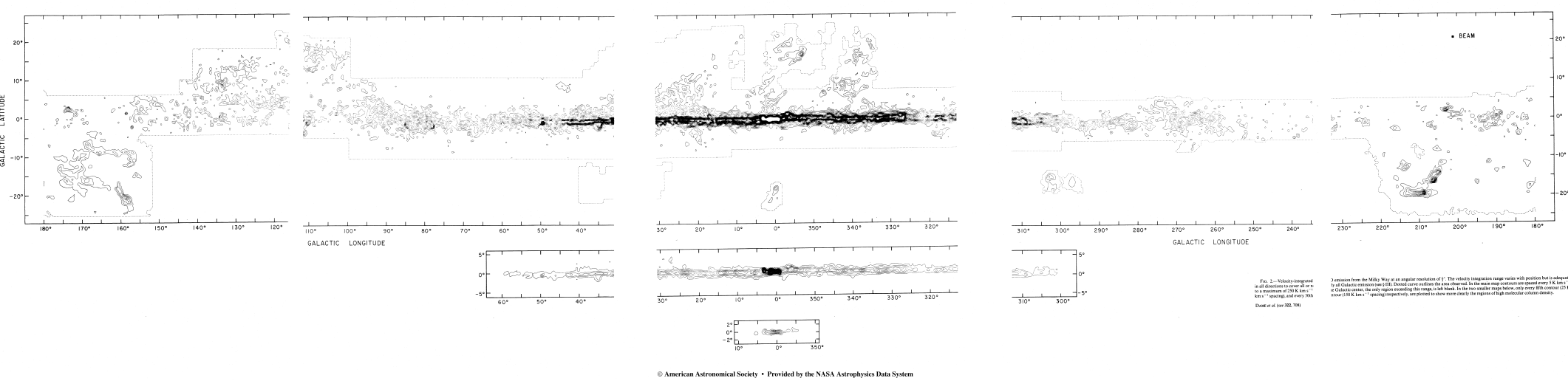
